\section{Estrutura de maternidades do Sistema Único de Saúde do Rio de Janeiro: desafio à qualidade do cuidado à saúde}

\section{The structure of Brazilian National Health Service Maternity Hospitals in Rio de Janeiro: the challenge of providing quality health care}

Cynthia Magluta 1

Marina Ferreira de Noronha 2

Maria Auxiliadora Mendes Gomes 3

Leda Amar de Aquino 4

Camila Aloísio Alves 5

Raulino Sabino da Silva 6

1,3,4 Instituto Fernandes Figueira/Fiocruz. Av Rui Barbosa, 716 Flamengo. CEP 20.250-020. Rio de Janeiro, RJ, Brasil. E-mail: cynthia@iff.fiocruz.br

2,6 Departamento de Administração e Planejamento em Saúde. Rio de Janeiro, RJ, Brasil.

5 Departamento de Gestão Hospitalar (DGH/SAS/MS). Ministério da Saúde. Rio de Janeiro, RJ, Brasil.

\begin{abstract}
Objectives: to evaluate the structure of Brazilian National Health Service (SUS) maternity hospitals in the State of Rio de Janeiro.

Methods: a normative cross-sectional evaluation was carried out of selected SUS maternity hospitals in the year 2005. Sixty-seven maternity hospitals were selected as a sample for reasons of convenience. Hospitals were observed and interviews conducted with health service managers and health workers. Various aspects of the structure of these services were examined, taking as a point of reference the norms provided by the Ministry of Health and outlined in the literature. These aspects included: installations, equipment, health team, training of health workers and availability of clinical guidelines.

Results: the presence of the structural requirements under evaluation varied according to the level of complexity, being less frequently found in maternity hospitals undertaking lower complexity procedures. The physical structure, availability of clinical guidelines and the presence of trained health workers in these services were more adequate than the training offered.

Conclusions: there is a need to invest more in professional training, to extend the availability of clinical guidelines, and to improve the physical structure of hospitals as a way of providing better quality perinatal care in conformity with national regulations.
\end{abstract}

Key words Perinatal care, Structure of services, Practice guidelines, Quality of health care

\section{Resumo}

Objetivos: avaliar a estrutura de maternidades pertencentes ao Sistema Único de Saúde (SUS) do Estado do Rio de Janeiro.

Método: realizou-se uma avaliação normativa, com corte transversal de maternidades selecionadas, vinculadas ao SUS no ano de 2005. Foram selecionadas 67 maternidades por amostragem de conveniência. Foram empregados um roteiro de observação e um de entrevistas com chefes de serviço e profissionais. Aspectos da estrutura destes serviços foram analisados, utilizando-se como referência a conformidade com as normas disponibilizadas pelo Ministério da Saúde e preconizadas na literatura. Esses aspectos foram: instalação, equipamentos, equipe de saúde, capacitação de profissionais $e$ disponibilidade de diretrizes clínicas.

Resultados: a presença dos requisitos da estrutura avaliados difere entre os estratos de complexidade, sendo menos frequente nas maternidades de menor complexidade. Os aspectos da estrutura física, disponibilidade de diretrizes clínicas e a presença dos profissionais nos serviços foram mais adequados do que a capacitação oferecida pelos serviços.

Conclusões: são necessários investimentos na capacitação dos profissionais, ampliação da disponibilidade de diretrizes clínicas e melhoria da estrutura física, visando qualificar o cuidado perinatal $e$ adequá-lo às regulamentações nacionais.

Palavras-chave Assistência perinatal, Estrutura dos serviços, Guias de prática clínica, Qualidade da assistência à saúde 


\section{Introdução}

A qualidade dos serviços de saúde e do cuidado em saúde depende de uma articulação complexa do processo de trabalho da equipe multidisciplinar com as condições de infraestrutura, dos serviços e do sistema de saúde, tendo a gestão um papel importante nesta articulação. ${ }^{1}$

Donabedian ${ }^{2}$ identifica que o cuidado em saúde é constituído por dois domínios: a aplicação dos conhecimentos e das tecnologias e a relação da equipe com o paciente. A qualidade desses dois domínios depende da estrutura como uma précondição favorável. É compreendida, pelo autor, como "as características relativamente estáveis dos provedores de cuidados, os instrumentos e recursos que eles têm disponíveis, o ambiente físico e organizacional no qual trabalham" incluindo diferentes aspectos. Pressupõe que uma boa estrutura dos serviços seja "provavelmente, o meio mais importante de proteção e promoção da qualidade do cuidado". ${ }^{2}$ Enfatiza, entretanto, que não há uma relação direta entre uma boa estrutura e a produção de cuidados de boa qualidade; uma estrutura inadequada pode reduzir as chances de um cuidado de qualidade, mas não é capaz de determinar este resultado.

No contexto do Sistema Único de Saúde (SUS), um dos desafios tem sido o de dotar os serviços das condições de estrutura adequada, pois essa é ainda instável. A falta de estrutura adequada pode comprometer a qualidade da atenção nos mais diversos serviços, inclusive naqueles do cuidado obstétrico e neonatal.3-4

Um dos elementos que compõem a estrutura dos serviços de saúde, segundo Donabedian, 2 é a organização dos profissionais, que inclui: o número e o perfil de qualificação, a disponibilidade de normas e diretrizes clínicas, presença de mecanismos de monitoramento da qualidade; manutenção e promoção da competência dos profissionais. Tamblyn e Battista 5 identificam que a adequação da qualificação dos profissionais à sua efetiva prática clínica, o monitoramento, com retorno da informação aos profissionais e as análises de casos clínicos melhoram a qualidade do cuidado.

Ainda com relação aos aspectos da estrutura, a utilização de diretrizes clínicas baseadas em evidências científicas tem sido apontada como uma ferramenta importante no esforço de melhorar a qualidade da assistência à saúde. Entretanto, a identificação de estratégias efetivas de disponibilização e promoção do cumprimento de tais diretrizes tem constituído um desafio para os gestores dos serviços. 6
A observação desse aspecto da estrutura é relevante para qualificar a assistência e atender ao direito à saúde.

Em relação à qualificação profissional no Brasil, a proposta de educação permanente dos profissionais de saúde vem ganhando destaque como estratégia de aprendizagem no trabalho que visam a transformação das práticas profissionais e sua adequação à realidade complexa e de rápida mudança dos serviços de saúde. 7,8

Partindo do pressuposto que a estrutura do serviço de saúde contribui para a melhoria da qualidade da assistência, este estudo tem como objetivo avaliar a estrutura de maternidades pertencentes ao Sistema Único de Saúde (SUS) do Estado do Rio de Janeiro, em conformidade com as normas estabelecidas pelo Ministério da Saúde do Brasil.

\section{Métodos}

Foi realizada uma avaliação normativa, ${ }^{9}$ com corte transversal de maternidades selecionadas no Estado do Rio de Janeiro, vinculadas ao SUS, no ano de 2005. Esta pesquisa integrou as ações gerenciais do Programa de Atenção Integral à Mulher, Criança e Adolescente da Secretaria de Saúde do Estado do Rio de Janeiro (PAISMCA/SES-RJ), voltadas à organização e qualificação do Sistema de Gestação de Alto Risco do Estado, sendo coordenada pelo Instituto Fernandes Figueira da Fundação Oswaldo Cruz (IFF/Fiocruz). Compartilhou-se da compreensão de que a avaliação é uma oportunidade de participação e construção do conhecimento que possibilita o aperfeiçoamento do sistema de saúde. 10

No período de estudo foram identificados os nascidos vivos de 146 maternidades vinculadas ao SUS no Estado do Rio de Janeiro. ${ }^{11}$ Entre estas, 63 (43\%) eram unidades públicas (gestão municipal, estadual ou federal), 77 (53\%), privadas conveniadas ao SUS e as universitárias, quer sejam de universidades públicas ou privadas, eram 6 (4\%).

Para esse estudo, os gestores do PAISMCA/SESRJ selecionaram as maternidades segundo os seguintes critérios de inclusão: maternidades com elevado volume de nascimentos vivos (NV) - cerca de 1000 $\mathrm{NV}$ por ano - serviços com vínculo de ensino e pelo menos uma maternidade de cada região do Estado. As maternidades selecionadas deveriam conter, em conjunto, mais de $50 \%$ de NV do Estado.

Das 67 maternidades selecionadas, 64 foram visitadas (3 estavam em obras, com a desativação do serviço de cuidado perinatal). Dentre estas, 34 (53\%) eram públicas, $25(39 \%)$ privadas conveniadas ao SUS e 5 (8\%) universitárias, correspondendo a $44 \%$ 
da oferta de serviços perinatais e responsáveis por $82 \%$ dos NV do Estado em 2005.12 Em 70\% delas ocorreram mais de $1000 \mathrm{NV}$. Todas as regiões do Estado foram representadas no estudo.

Foi utilizada uma classificação de complexidade desenvolvida por Schramm 12 e adaptada pela equipe de pesquisadores. A classificação de Schramm 12 indica quatro níveis de complexidade de hospitais segundo a existência ou não de terapia intensiva para o adulto e para o neonato e considera, ainda se o hospital é ou não universitário. Para este estudo utilizou-se os quatro níveis de classificação segundo existência de terapia intensiva, acrescentando dois níveis de presença ou não de terapia semi-intensiva neonatal e um nível de terapia intensiva para adultos somente.

Para caracterizar-se uma unidade de cuidado neonatal, verificou-se ainda se ela dispunha de pelo menos cinco leitos, atendendo a uma portaria do Ministério da Saúde13 com os equipamentos para o cuidado neonatal adequados para o número de leitos (incubadoras, berços de calor radiante, monitores e respiradores). A classificação adaptada contém cinco níveis, a saber: I - Maternidade sem serviço de terapia intensiva de adulto ou neonatal; IIM - Maternidade com serviço de terapia intensiva de adulto; IIC UI - Maternidade com serviço de terapia semiintensiva neonatal - unidade intermediária; IIC Maternidade com serviço de terapia intensiva neonatal; III - Maternidade com serviço de terapia intensiva de adulto e terapia intensiva neonatal.

Duas fontes de informações foram utilizadas para classificar as maternidades: Cadastro Nacional de Estabelecimentos de Saúde (CNES) e aqueles obtidos durante a avaliação no local, prevalecendo este último.

A coleta dos dados foi realizada utilizando dois instrumentos adaptados da pesquisa realizada pelo PAISMCA/SES-RJ em 1997. Um questionário, contendo questões estruturadas e semiestruturadas para entrevista com os gestores da instituição (diretor e chefe de serviço) e com os profissionais: médicos e enfermeiros. As perguntas eram diferenciadas para cada um dos interlocutores, os quais eram entrevistados em momentos distintos. Para esse artigo utilizou-se o que os médicos e enfermeiros responderam sobre sua qualificação profissional, mais especificamente, quanto à participação em congressos e em atividades de qualificação oferecidas pelo serviço e o que os gestores informaram quanto à disponibilidade de diretrizes clínicas.

O segundo instrumento foi um roteiro de observação dos locais de assistência direta à mulher e ao recém-nascido, contendo aspectos da estrutura física e da disponibilidade de profissionais e equipamentos críticos para o cuidado obstétrico e neonatal.

As entrevistas e a observação foram realizadas por profissionais com experiência na área obstétrica, neonatal e de gestão de programa materno-infantil, treinados para esta pesquisa, no período de novembro de 2005 a fevereiro de 2006. Os instrumentos de coleta de dados continham instruções para seu preenchimento, visando minimizar a variação entre os observadores. Foi realizado um teste piloto do questionário por uma das equipes de pesquisadores de campo, cujos resultados foram incluídos no treinamento dos avaliadores. Para realizar o trabalho de campo, foram constituídas dez equipes compostas por dois profissionais.

Os aspectos da estrutura utilizados no estudo foram selecionados dentre aqueles explicitados nas normas disponibilizadas pelo Ministério da Saúde brasileiro cujos padrões de referência utilizados estão contidos na RDC 50,14 na portaria MS/GM 56915 e na Política Nacional de Educação Permanente 7 atendendo aos seguintes critérios: que estes requisitos da estrutura fossem obrigatórios, independente da complexidade da maternidade, que fossem relevantes para a segurança da mulher e do recémnascido para o momento do parto.

Dentre os requisitos de estrutura, exigidos na RDC 5014 e aplicáveis a enfermaria foi selecionado a disponibilidade de pia e a existência de um berço para cada leito materno. Em relação ao centro cirúrgico foram selecionados os seguintes aspectos: disponibilidade de pontos de oxigênio, de mesas de parto e mesa cirúrgica, focos cirúrgicos, carros de emergência com desfibrilador para ressuscitação da mulher e equipamento para ressuscitação do recém nascido (berço de calor radiante). Dos itens previstos na portaria MS/GM 56915 foram selecionados: a disponibilidade de instrumentais cirúrgicos e materiais de ressuscitação para o recém-nascido (laringoscópio e cânula endotraqueal).

Adicionalmente os autores consideraram para a estrutura da enfermaria a disponibilidade de ar condicionado como forma de climatização, que é uma recomendação e não uma exigência da RDC 50.14

A portaria MS/GM 56915 também indica quais profissionais devem estar presentes para o cuidado da mulher e do recém nascido, especificando ainda sua qualificação. Para o cuidado obstétrico está previsto que os médicos tenham esta especialidade e para o cuidado neonatal podem ser pediatras ou neonatologistas. Em relação aos enfermeiros a especialização em obstetrícia é um requisito desejável.

Considerou-se como "profissionais presentes" 
aqueles que estavam em atividade no momento da visita. Reportou-se como "qualificação adequada" quando a especialidade do profissional correspondia àquela de seu exercício profissional e estava em conformidade com a Portaria MS/GM 569.15

Verificou-se a participação dos profissionais em atividades de capacitação no último ano, compreendendo ser esta uma das responsabilidades da gestão dos serviços, conforme a Política Nacional de Educação Permanente em Saúde. ${ }^{7}$ Adicionalmente, verificou-se a busca de outras oportunidades de capacitação através da participação em congressos, financiados pelos serviços ou por eles próprios. A participação em congressos foi incluída no estudo, uma vez que é uma das formas usuais de atualização dos profissionais e que foi considerada uma boa prática pelos autores.

Foi verificada, junto aos gestores da instituição, a disponibilidade de algumas das diretrizes clínicas do cuidado obstétrico recomendadas pelo MS, a saber: pré-eclampsia e eclampsia, 16 corticóide antenatal em trabalho de parto prematuro, 17 rastreamento de sífilis e de HIV/AIDS.15 Quanto às diretrizes para o cuidado neonatal, foram utilizadas aquelas referidas na literatura especializada sobre parâmetros para o cuidado neonatal 18,19: ter surfactante definido, ter antibiótico de primeira linha definido e o manejo da dor do recém-nascido.

Quanto à forma de disseminação das diretrizes clínicas, somente os gestores das unidades de complexidade IIC e III responderam fazer uso de diretrizes clínicas em seus serviços e, portanto, foram estes que puderam responder sobre as formas de disseminação. As formas de divulgação mencionadas foram: por escrito em livros ou pastas, afixadas em murais dos serviços e divulgadas em reuniões dos serviços. Foi considerada uma ou várias formas de divulgação, sendo esta última forma incluída como uma categoria na análise.

Os dados coletados foram digitados no programa de processamento de dados SPSS, versão 12. Foram realizadas análises descritivas de todos os aspectos da estrutura dos serviços observadas na avaliação local das maternidades. Para este trabalho, as análises foram realizadas a partir da classificação das maternidades em graus de complexidade. Foram apresentadas características gerais das maternidades como: localização, natureza jurídica, número de leitos. Seguiu-se com a apresentação da proporção das maternidades que tinham disponibilidade, no momento da avaliação local de cada maternidade, dos aspectos selecionados da estrutura tais como: instalações físicas e disponibilidade de equipamentos; de instrumentais e materiais; de profissionais e das dire- trizes clínicas.

Foi considerada como adequada, a estrutura das maternidades que estava em conformidade com os aspectos selecionados das normas brasileiras (RDC 5014 e Portaria MS/GM 56915). Foi também apresentada a análise das maternidades que apresentavam este conjunto de características da estrutura para a atenção à mulher e para o recém-nascido, representando um indicador síntese da qualidade da estrutura dos serviços avaliados.

O projeto foi aprovado pelo Comitê de Ética em Pesquisa em seres humanos do IFF/FIOCRUZ sob o $n^{\circ}$ CAAE 0259.0.008.000-05 em 14/09/2005. Foram solicitados consentimentos informados da SES/RJ, dos diretores de cada maternidade e de cada mulher e profissional que participou da pesquisa, sendo preservadas as identidades de profissionais e pacientes.

\section{Resultados}

A distribuição das maternidades estudadas encontrase na Tabela 1.

A maior proporção das 64 maternidades visitadas (59\%) foi do município do Rio de Janeiro e das regiões metropolitanas I e II.

Cerca de $25 \%$ das maternidades foram classificadas como de alta complexidade (nível III) e semelhante percentual como baixo nível de complexidade (nível I). Metade das maternidades visitadas era pública e $39 \%$ privadas conveniadas ao SUS. Das maternidades públicas, $15 \%$ eram de menor complexidade (nível I), enquanto em relação às privadas quase metade (48\%) eram desta complexidade como se pode observar na Tabela 1. Do total de nascimentos destas maternidades, a maior proporção ocorreu em maternidades com disponibilidade de UTI Neonatal (25\% na complexidade IIC e $35 \%$ na complexidade III) e $25 \%$ dos nascimentos ocorreu em maternidades com UI neonatal.

Quanto à presença dos requisitos da estrutura observados, verificou-se variação tanto entre maternidades de diferentes graus de complexidades como entre os itens avaliados (Tabela 2). Em menos de $50 \%$ das maternidades, observou-se a presença de pia e de climatização com ar condicionado nas enfermarias. A disponibilidade de pontos de oxigênio foi observada em $95 \%$ das maternidades.

O carro para emergência com desfibrilador foi encontrado em todas as maternidades da complexidade IIC e em cerca de $80 \%$ das maternidades dos outros estratos. Cabe ressaltar que mesas de parto, mesas cirúrgicas e foco cirúrgico foram encontrados em todas as maternidades.

A disponibilidade de instrumental cirúrgico 
Distribuição da localização, natureza jurídica e leitos das maternidades segundo grau de complexidade. Estado do Rio de Janeiro, 2005-2006.

\begin{tabular}{|c|c|c|c|c|c|c|}
\hline \multirow[t]{2}{*}{ Itens } & \multicolumn{5}{|c|}{ Complexidade } & \multirow[t]{2}{*}{ Total } \\
\hline & I & II M & II C UI & II C & III & \\
\hline Número de Maternidades & 17 & 13 & 8 & 10 & 16 & 64 \\
\hline \multicolumn{7}{|l|}{ Região do Estado } \\
\hline Município Rio de Janeiro & 0 & 3 & 2 & 6 & 6 & 17 \\
\hline Metropolitana I & 7 & 1 & 2 & 1 & 3 & 14 \\
\hline Metropolitana II & 2 & 1 & 1 & 1 & 2 & 7 \\
\hline Baía da Ilha Grande & 1 & 0 & 1 & 0 & 0 & 2 \\
\hline Médio Paraíba & 2 & 1 & 0 & 2 & 1 & 6 \\
\hline Centro Sul Fluminense & 0 & 1 & 0 & 0 & 1 & 2 \\
\hline Serrana & 1 & 2 & 1 & 0 & 1 & 5 \\
\hline Baixada Litorânea & 3 & 1 & 1 & 0 & 0 & 5 \\
\hline Noroeste & 1 & 1 & 0 & 0 & 0 & 2 \\
\hline Norte & 0 & 2 & 0 & 0 & 2 & 4 \\
\hline \multicolumn{7}{|l|}{ Natureza Jurídica } \\
\hline Públicas & 5 & 6 & 6 & 7 & 10 & 34 \\
\hline Conveniadas & 12 & 6 & 2 & 2 & 3 & 25 \\
\hline Universitárias & 0 & 1 & 0 & 1 & 3 & 5 \\
\hline \multicolumn{7}{|l|}{ Número de Leitos } \\
\hline Obstétricos & 231 & 60 & 383 & 459 & 705 & 1838 \\
\hline Neonatais & 10 & 19 & 58 & 231 & 322 & 640 \\
\hline UTI Adulto & 3 & 18 & 4 & 3 & 182 & 210 \\
\hline
\end{tabular}

variou de $60 \%$ para o estrato IIC a $88 \%$ na complexidade I. Quanto à existência dos equipamentos e materiais para ressuscitação neonatal, todas as maternidades de complexidade IIC e III dispunham de tais recursos.

A presença de profissionais médicos ou enfermeiros para o cuidado à mulher foi verificada em $81 \%$ das maternidades de complexidade III e em todas as de complexidades IIC UI e IIC. Os pediatras/neonatologistas foram encontrados no momento da avaliação em $100 \%$ das maternidades de complexidade IIC UI, enquanto que em somente $69 \%$ daquelas de complexidade IIM.

Durante a visita também foram verificadas condições de estrutura para a UTI Neonatal, que não está descrita na Tabela 2 por referir-se somente às maternidades de nível IIC e III. Entre os aspectos observados, identificou-se que em metade das UTI Neonatais existiam pia, climatização e limpeza adequada, e em $38 \%$ dos serviços havia condições para a permanência da família junto aos recém-nascidos.

Em $90 \%$ das maternidades o obstetra ou enfer- meiro estava presente, e em $86 \%$ o pediatra. Entre os profissionais presentes, observou-se que a maioria é especialista na sua área de atuação e que esta corresponde ao que está indicado na Portaria MS/GM 569,15 sendo, portanto considerada uma qualificação adequada. Os médicos em sua maioria eram formados em obstetrícia ( $89 \%$ ) ou pediatria (75\%), tendo em alguns poucos casos, profissionais com outra formação. Nesta última situação foi verificada a presença de cirurgião geral atuando como obstetra e otorrinolaringologista e alergista realizando o cuidado de recém-nascidos. Os enfermeiros tinham a qualificação adequada em 53\% das maternidades.

A participação em capacitações oferecidas pelo serviço no último ano foi mencionada por menos de $40 \%$ dos obstetras e pediatras entrevistados e por $53 \%$ dos enfermeiros. Mencionaram que estas capacitações eram de curta duração variando entre 20 e 40h. Para os obstetras, as capacitações versavam sobre as principais causas de mortalidade materna: hipertensão arterial (8\%), hemorragia (5\%), infecção $(5 \%)$ e abortamento $(6 \%)$.Os pediatras mencionaram 
Distribuição dos aspectos da estrutura e qualificação dos profissionais das maternidades segundo grau de complexidade. Estado do Rio de Janeiro, 2005-2006.

Aspectos da estrutura e qualificação dos profissionais

Complexidade

\begin{tabular}{cccccc}
\hline I & II M & IIC UI & IIC & III & Total \\
\hline $\mathrm{na}^{\mathrm{a}=17}$ & $\mathrm{n}=13$ & $\mathrm{n}=8$ & $\mathrm{n}=10$ & $\mathrm{n}=16$ & $\mathrm{n}=64$
\end{tabular}

Enfermaria

Climatização com ar condicionadob

Disponibilidade de piac

Berço para cada leito maternoc

Centro Obstétrico

Ponto de oxigênio

Equipamentos de ressuscitação da mulherc

Disponibilidade de instrumental cirúrgico no último mêsd

Equipamentos de ressuscitação recém-nascidod

Disponibilidade de material ressuscitação recém-nascidod

Profissionais presentesd

Obstetras ou Enfermagem obstétrica

Pediatras/Neonatologistas

Qualificação adequadad

Obstetras

Pediatras/Neonatologistas

Enfermagem obstétrica

Capacitação no último anoe

\section{Obstetras}

Pediatras/Neonatologistas

Enfermagem obstétrica

Participação em Congressob

Financiado pelo serviço

Obstetras

Pediatras/Neonatologistas

Enfermagem obstétrica

Financiado pelo profissional

Obstetras

Pediatras/Neonatologistas

Enfermagem obstétrica

$\begin{array}{rr}35 & 38 \\ 41 & 46 \\ 82 & 100 \\ & \\ 82 & 100 \\ 76 & 85 \\ 88 & 69 \\ 71 & 100 \\ 76 & 92\end{array}$

50

25

25
88

60

50

31

62

40

$100 \quad 100$

47

94

100

100

100

95

$100 \quad 81 \quad 84$

$\begin{array}{lll}60 & 69 & 73\end{array}$

$100 \quad 100 \quad 91$

$\begin{array}{lll}100 & 100 & 92\end{array}$

$\begin{array}{llllll}88 & 92 & 100 & 100 & 81 & 90\end{array}$

$\begin{array}{llllll}82 & 69 & 100 & 90 & 94 & 86\end{array}$

$\begin{array}{llllll}82 & 77 & 100 & 90 & 100 & 89\end{array}$

$53-77 \quad 75$

100

81

50

100

56

53

$\begin{array}{llllll}35 & 31 & 50 & 30 & 44 & 38\end{array}$

$\begin{array}{llllll}12 & 38 & 38 & 40 & 44 & 32\end{array}$

$41 \quad 38$

75

80

63

32

56

$\begin{array}{rrrrrr}12 & 0 & 0 & 10 & 25 & 11 \\ 0 & 0 & 0 & 0 & 0 & 0 \\ 6 & 8 & 0 & 10 & 13 & 8 \\ 47 & 46 & 50 & 60 & 56 & 52 \\ 29 & 38 & 75 & 80 & 50 & 50 \\ 12 & 23 & 13 & 20 & 31 & 20\end{array}$

Referência do item: a= número de maternidades do estrato de complexidade, b=boa prática; c= RDC 50; $d=$ Portaria MS/GM 569; e = Política Nacional de Educação Permanente.

capacitações nos temas: a implantação da Estratégia Hospital Amigo da Criança (11\%), teste do reflexo vermelho (8\%), DST e AIDS (3\%) e reanimação neonatal (5\%). Dentre os enfermeiros, $28 \%$ relatou a participação em capacitações voltadas para o estímulo ao aleitamento materno e a implantação da Estratégia Hospital Amigo da Criança.
A participação em congressos financiada pelos hospitais foi referida por cerca de $10 \%$ dos obstetras e enfermeiros, tendo se concentrado em maternidades de maior complexidade (níveis IIC e III). Já a participação em congressos financiada pelo próprio profissional foi mencionada por aproximadamente metade dos médicos (obstetras e pediatras) e $20 \%$ 
dos enfermeiros de unidades de maior complexidade (IIC e III).

Verificou-se que $60 \%$ das maternidades de complexidade IIC UI e IIC estão em conformidade com todos os requisitos, enquanto nos outros estratos de complexidade, metade ou menos da metade das maternidades estão em conformidade. Observou-se que as maternidades apresentaram melhor qualidade da estrutura para o cuidado ao recém-nascido (78\%) do que para o cuidado da mulher (55\%) (Tabela 3$)$.
As diretrizes relativas (Tabela 4) ao cuidado obstétrico foram relatadas como disponíveis, variando de $75 \%$ a $100 \%$ em todos os estratos de complexidade, exceto no nível I, no qual 53\% a $77 \%$ das maternidades reportaram ter tais diretrizes. As diretrizes para o cuidado neonatal são requisitos somente para as unidades de complexidade IICUI, IIC e III; entre estas o manejo da dor é a diretriz que está menos disponível nas maternidades de complexidade IICUI (38\%) e nas de nível III (63\%).

\section{Tabela 3}

Distribuição das maternidades por grau de complexidade, segundo presença de aspectos selecionados da estrutura, para o cuidado à mulher e ao recém-nascido. Estado do Rio de Janeiro, 2005-2006.

\section{Aspectos da estrutura}

Complexidade

Total

\begin{tabular}{|c|c|c|c|c|c|c|c|c|c|c|c|}
\hline \multicolumn{2}{|c|}{ I } & \multicolumn{2}{|c|}{ II M } & \multicolumn{2}{|c|}{ II C UI } & \multicolumn{2}{|c|}{ II C } & \multicolumn{2}{|c|}{ III } & & \\
\hline$n$ & $\%$ & $\mathrm{n}$ & $\%$ & $n$ & $\%$ & $\mathrm{n}$ & $\%$ & $\mathrm{n}$ & $\%$ & $n$ & $\%$ \\
\hline
\end{tabular}

Cuidado à mulher que inclui a presença de ponto de oxigênio, carro de emergência para ressuscitação da mulher e instrumental cirúrgico no centro obstétrico $\begin{array}{llllllllllll}10 & 59,0 & 7 & 54,0 & 5 & 63,0 & 6 & 60,0 & 7 & 44,0 & 35 & 55,0\end{array}$ e presença do obstetra ou enfermeiro na maternidade

Cuidado ao RN que inclui ponto de oxigênio, berço de calor radiante, material para ressuscitação do RN no centro obstétrico e presença do pediatra/neonatologista na maternidade

Todos os critérios de estrutura para o cuidado à mulher e ao RN

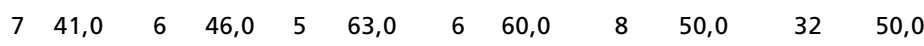

$\mathrm{RN}=$ recém-nascido.

Tabela 4

Distribuição das maternidades por grau de complexidade e segundo disponibilidade de diretrizes clínicas para o cuidado obstétrico e neonatal. Estado do Rio de Janeiro, 2005-2006.

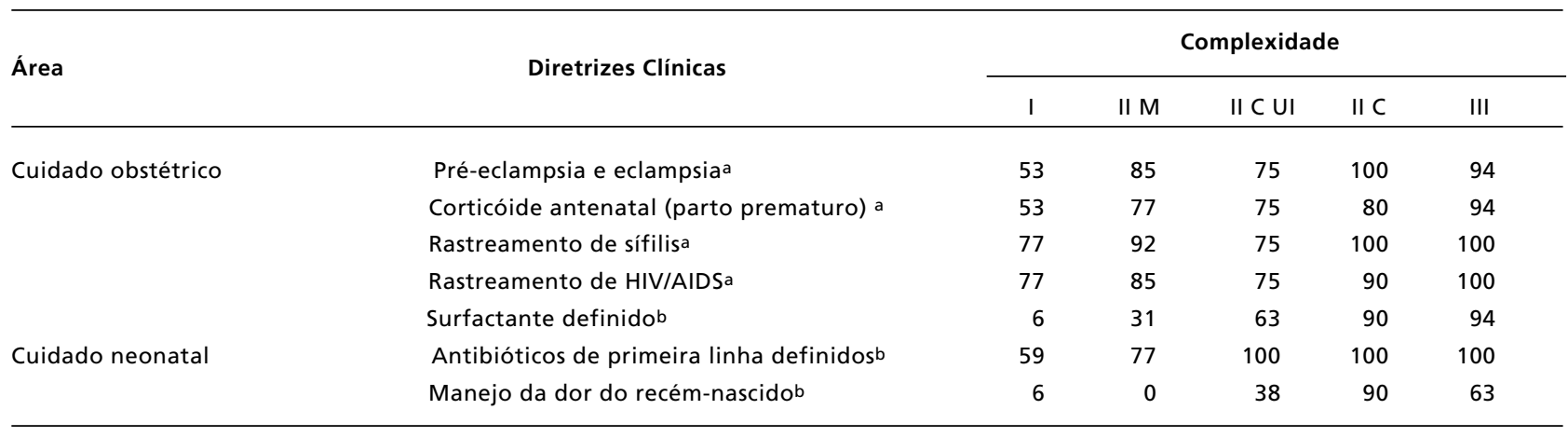

$a$ = informação obtida com o chefe da maternidade; b = informação obtida com o chefe da neonatologia; 
As estratégias de divulgação das diretrizes clínicas foram descritas somente para os níveis IIC e III por serem os que mais reportaram seu uso. A estratégia mais frequente foi a disponibilização das diretrizes por escrito, organizada em um livro ou pasta. Os chefes dos serviços reportavam que as mesmas estavam disponíveis para os profissionais durante o trabalho de rotina ou plantão, tendo sido confirmado pelos plantonistas. Utilizar reuniões para divulgar as diretrizes é referido por menos da metade dos serviços de neonatologia e cerca de $20 \%$ nos de obstetrícia. Metade dos serviços de nível IIC reportou mais de uma estratégia de divulgação e também cerca de $30 \%$ dos serviços de nível III (Tabela 5).

Tabela 5

Distribuição das maternidades por especialidade obstétrica e neonatal, pelos graus de complexidade IIC e III, segundo estratégias mencionadas pelos chefes de serviços de obstetrícia e neonatologia, para divulgação de diretrizes clínicas. Estado do Rio de Janeiro, 2005-2006.

Estratégia utilizada para divulgação da diretriz clínica

Especialidade

\begin{tabular}{|c|c|c|c|c|}
\hline & & & & \\
\hline & \multicolumn{2}{|c|}{ Obstétrica } & \multicolumn{2}{|c|}{ Neonatal } \\
\hline & \multicolumn{2}{|c|}{ Complexidade } & \multicolumn{2}{|c|}{ Complexidade } \\
\hline & IIC & III & II C & III \\
\hline Mural & 50 & 25 & 50 & 6 \\
\hline Escrito & 90 & 69 & 60 & 38 \\
\hline Reunião clínica & 20 & 25 & 30 & 44 \\
\hline Mais de uma estratégia & 50 & 31 & 60 & 38 \\
\hline
\end{tabular}

\section{Discussão}

A infra-estrutura das maternidades mostrou-se variável pelos estratos de complexidade e segundo os diversos requisitos de estrutura selecionados nas normas brasileiras aplicáveis nesta área de atenção à saúde. Foram identificados comprometimentos na estrutura em termos de instalação física, disponibilidade de equipamentos, instrumentais cirúrgicos, materiais e presença dos profissionais, quando analisados em separados ou em conjunto.

A configuração tradicional (mesas de parto, cirúrgicas e focos) de um centro obstétrico foi encontrada em todas as maternidades, no entanto a disponibilidade de equipamento para ressuscitação da mulher, uma exigência da RDC 50,14 foi somente encontrada nas maternidades de complexidade IIC.

Em somente $73 \%$ das maternidades foi evidenciada a disponibilidade de instrumental cirúrgico, item fundamental dos ambientes cirúrgicos, mas que depende do processo eficiente de esterilização, manutenção e compra de novos itens para reposição. $\mathrm{O}$ desempenho do conjunto de maternidades neste aspecto pode indicar a necessidade de que os processos gerenciais sejam aprimorados. Chama a atenção que
$88 \%$ das maternidades da complexidade I atenderam a este requisito e são em sua maioria $(70 \%)$ privadas conveniadas ao SUS, sendo este o único item que este estrato sobressaiu positivamente.

A concepção de Donabedian2 de que a estrutura é uma característica relativamente estável dos serviços de saúde não parece ter sido alcançada pelo conjunto de maternidades avaliadas e que são as maiores disponíveis no Estado do Rio de Janeiro.

A configuração do centro obstétrico para a ressuscitação neonatal tem sido alvo de ação governamental, que instituiu no SUS, em 1993, a valorização da qualidade da assistência imediata ao recém-nascido, com a previsão de ressarcimento pelo SUS da presença do pediatra na sala de parto. ${ }^{20} \mathrm{O}$ programa de qualificação em ressuscitação neonatal para pediatras desenvolvido pela Sociedade Brasileira de Pediatria (SBP), representada no Estado pela Sociedade de Pediatria do Rio de Janeiro, pode também ter contribuído para o resultado observado. ${ }^{21}$

A SBP considera que "a ressuscitação do recémnascido na sala de parto é um dos mais importantes desafios na prática pediátrica, pois terá repercussões por toda a vida definindo a qualidade de vida do bebê, da família e o papel deste na sociedade”. Apre- 
senta em seu sítio na internet, 21 a estimativa da Academia Americana de Pediatria, segundo a qual de 5 a $10 \%$ de todos os bebês necessitam de alguma manobra de ressuscitação ao nascimento e de 1 a $10 \%$ dos nascidos em hospitais precisam de ventilação com pressão positiva.

No momento da visita às maternidades, verificou-se que em $10 \%$ delas estavam ausentes obstetras ou enfermeiros e em $14 \%$ os pediatras. A presença desses profissionais é prevista em portaria do MS15 e seu não cumprimento coloca em risco a vida da mulher e da criança.

Para a maioria das maternidades de maior complexidade, a qualificação dos profissionais se mostrou adequada somente para as maternidades de maior grau de complexidade. Este aspecto da estrutura é considerado um requisito básico para a qualidade da assistência 2,5 e se mostrou adequada por corresponder ao que está previsto na referência normativa utilizada (Portaria MS/GM 56915). A participação em capacitações oferecidas pelos serviços e/ou pelas secretarias de saúde foi menor para os médicos (menos de $40 \%$ ) do que para os enfermeiros (53\%). A maior parte das capacitações foi centrada no aleitamento materno, que embora seja um tema pertinente e relevante ao cuidado perinatal é insuficiente para as necessidades do cotidiano desses serviços. Uma hipótese para tentar explicar este resultado pode ser a forma e/ou conteúdo inadequados de capacitação para as categorias profissionais, não conseguindo atrair o profissional. Verificou-se que a oferta das capacitações eram através de cursos e exposições teóricas, estratégias de menor impacto para a mudança da prática profissional.6,8 Neste sentido, a proposta de educação permanente, que implica em um cotidiano reflexivo, com diversas oportunidades de capacitação e com vistas a transformar as práticas profissionais 7 , pode vir a responder às dificuldades e deficiências na área.

Como um dos aspectos da estrutura dos serviços de saúde, o sucesso da implementação das diretrizes clínicas depende de um esforço organizado dos serviços e dos gestores do sistema de saúde. 6 A menção da disponibilidade de diretrizes clínicas para o cuidado obstétrico e neonatal variou entre os estratos de complexidade e segundo a diretriz investigada. A menor disponibilidade de diretrizes como o rastreamento de sífilis e HIV/AIDS15 é paradoxal, pois essas são questões de alta relevância epidemiológica, abordadas em diversos programas governamentais ao longo dos anos.

Somente disponibilizar as diretrizes clínicas tem sido reportado como insuficiente para qualificar a prática assistencial. $6 \mathrm{Um}$ estudo sobre as práticas para prevenção da broncodisplasia pulmonar em recém-nascidos de muito baixo peso ao nascer, realizado em uma UTI neonatal pública, localizada no município do Rio de Janeiro, evidenciou que os médicos mencionam corretamente algumas práticas, tais como: uso criterioso de oxigênio $(86,7 \%)$ e o uso do surfactante precoce $(71,1 \%)$. No entanto, quando a presença desses procedimentos foi verificada nos prontuários, sua frequência reduziu para $13 \% \mathrm{e}$ $38,7 \%$ respectivamente. 22

Boas condições de estrutura são consideradas pré-condição favorável à melhoria da qualidade do cuidado e das práticas profissionais, possibilitando que no cotidiano dos serviços, as melhores práticas apoiadas em evidências científicas sejam sempre utilizadas em benefício dos pacientes. Estas estão ligadas à estruturação do sistema de saúde, são mais dependentes das decisões dos gestores, mas também são influenciadas pelas ações da equipe de atenção, quando demandam condições adequadas de estrutura. 2,5

$\mathrm{Na}$ busca de ferramentas para uma gestão de qualidade, a participação da equipe de atenção nas decisões dos serviços de saúde é defendida por diversos autores tanto no contexto da gestão de serviços de saúde, educação permanente, quanto na Política Nacional de Humanização. 23 No cotidiano dos serviços, tais proposições podem se traduzir em espaços colegiados, apoiados por diversos tipos de profissionais, como os apoiadores matriciais propostos por Campos. 24 Estas proposições encontram semelhanças para duas estratégias estudadas por Grimshaw et al.6: a análise da prática a partir da informação dos resultados da atenção e a assessoria de especialistas para discutir diretrizes clínicas e/ou aspectos dos serviços.

O trabalho da Vermont Oxford Network, ${ }^{25}$ uma rede colaborativa norte-americana de UTI neonatais, pode ser referido como um exemplo nesta busca. Os participantes dessa rede observaram que estar em equipe nas UTI Neonatais não é o mesmo que realizar um trabalho de equipe, coordenado e fruto de consenso. Portanto, nessa rede, são utilizadas ferramentas da gestão pela qualidade e é desenvolvido um trabalho de longo prazo de qualificação dos profissionais, adaptação de diretrizes, busca ativa das possíveis melhores práticas entre os filiados à rede $\mathrm{e}$ realização de análises comparativas dos resultados do cuidado neonatal, com retorno aos serviços e profissionais.

Verificou-se, portanto, que as condições da estrutura das maternidades ainda podem ser consideradas um desafio, não parecem ser estáveis, ${ }^{2}$ havendo variações quanto à presença de itens previstos na regula- 
mentação sanitária brasileira ${ }^{14,15}$ e que independem da complexidade da maternidade. Itens esses também recomendados desde 1987, pela Organização Pan-americana de Saúde (OPAS) em instrumento destinado à avaliação de serviços materno-infantis que incluía os aspectos relativos às maternidades. ${ }^{26}$

Análise das relações entre a estrutura, o processo de cuidado e os resultados não são objetivos do presente estudo, embora sejam importantes uma vez que há estudos que demonstram resultados indesejáveis, como a mortalidade evitável de recémnascidos prematuros 27 e a peregrinação ante-natal 28 ocorrendo no Estado do Rio de Janeiro.

Aliado aos esforços de melhoria da estrutura, cabe aos gestores, às organizações acadêmicas e às equipes dos serviços de saúde desenvolver mecanismos que ampliem sua comunicação, interação, promovendo o aprender a aprender, visando contribuir para que o cotidiano da prestação do cuidado

\section{Referências}

1. Novaes HMD. Pesquisa em, sobre e para os serviços de saúde: panorama internacional e questões para a pesquisa em saúde no Brasil. Cad Saúde Pública. 2004; 20 (Supl 2): S147-173.

2. Donabedian A. The definition of quality: approaches to its assessement. Ann Arbor: Health Administration Press; 1980.

3. Gomes MAM. Organização da Assistência Perinatal no Brasil. In: Maria Elizabeth Lopes Moreira, José Maria de Andrade Lopes e Manoel de Carvalho, organizadores. O recém-nascido de alto risco: teoria e prática do cuidar. Rio de Janeiro: Editora Fiocruz; 2004. p. 21-48.

4. Lansky S, França E, César CC, Neto LCM, Leal MC. Mortes perinatais e avaliação da assistência ao parto em maternidades do Sistema Único de Saúde em Belo Horizonte, Minas Gerais, Brasil, 1999. Cad Saúde Pública. 2006; 22: 117-30.

5. Tamblyn R, Battista R. Changing clinical practice: which interventions work? J Contin Educ Health Prof. 1999; 13: 273-88.

6. Grimshaw J, Thomas R, MacLennan G, Fraser C, Ramsay CR, Vale L, Whitty P, Eccles MP, Matowe L, Shirran L, Wensing M, Dijkstra R. Effectiveness and efficience of guideline dissemination and implementation strategies. Health Technol Assess. 2004; 8: 1-72.

7. Brasil. Portaria GM/MS 1.996, de 20 de agosto de 2007, dispõe sobre as diretrizes para a implementação da Política Nacional de Educação Permanente. Publicada no Diário Oficial da União $n^{\circ} 162$, de 22 de agosto de 2007.

8. Ceccim RB. Educação Permanente em saúde: desafio ambicioso e necessário. Interface Comun Saúde Educ. 2005; 9 : 161-77. seja de qualidade, favorecendo, portanto, o cumprimento do direito que os brasileiros conquistaram no texto constitucional, de um cuidado acolhedor e com aplicação prudente dos conhecimentos e das técnicas.

Esta pesquisa foi financiada pela Secretaria de Saúde do Estado do Rio de Janeiro como parte de suas ações de implantação do Sistema Estadual de Atenção à Gestação de Alto Risco.

\section{Agradecimentos}

Agradecemos às Dras. Tizuko Shiraiwa e Eliana Calasans, coordenadoras do Programa de Atenção Integral à Saúde da Mulher, Criança e Adolescente, pelo apoio técnico e efetivo ao desenvolvimento da pesquisa e pelo financiamento concedido. Agradecemos ainda o empenho de todos os pesquisadores de campo.
9. Contandriopoulos AP, Champanhe F, Denis JL, Pineault R. A avaliação na área da saúde: conceitos e métodos, in Zulmira Maria Araújo Hartz (org). Avaliação em saúde: dos modelos conceituais à prática na análise da implantação de programas. Rio de Janeiro: Ed. Fiocruz; 1997. p. 29-47.

10. Takeda S, Talbot Y. Avaliar, uma responsabilidade. Ciênc Saúde Coletiva. 2006; 11: 564-76.

11. Sistema Integrado de Nascimentos Vivos (SINASC) acessado através de CD-Room cedido pelo Departamento de Dados Vitais da Secretaria de Estado de Saúde do Rio de Janeiro; 2005.

12. Schramm JMA, Szwarcwald CL, Esteves MAP. Assistência obstétrica e risco de internação na rede de hospitais do Estado do Rio de Janeiro. Rev Saúde Pública. 2002; 36 : 590-7.

13. Brasil. Portaria 3.432 GM de 12 de agosto de 1998. Estabelece critérios de classificação para as unidades de tratamento intensivo - UTI. Diário Oficial da União de 12 de agosto de 1998.

14. Brasil. RDC 50, de 21 de fevereiro de 2002, dispõe sobre o regulamento técnico para o planejamento, programação, elaboração e avaliação de projetos físicos de estabelecimentos de saúde. Diário Oficial da União de 20 de março de 2002.

15. Brasil. Portaria MS/GM 569, de 1 de Junho de 2000, institui o Programa de Humanização no Pré-Natal e Nascimento, no âmbito do Sistema Único de Saúde. Diário Oficial da União de 8 de junho de 2000 .

16. Brasil. Ministério da Saúde. Urgências e Emergências maternas: guia para diagnóstico e conduta em situações de risco de morte materna. Brasília; 2000. 
17. Brasil. Ministério da Saúde. Parto, aborto, e puerpério: assistência humanizada à mulher. Brasília; 2001.

18. Moreira MEL. Praticando a neonatologia baseada em evidências. In: Maria Elisabeth Lopes Moreira, José Maria de Andrade Lopes e Manoel de Carvalho (org). O recémnascido de alto risco: teoria e prática do cuidar. Rio de Janeiro: Ed. Fiocruz; 2004.

19. Sharek PJ, Baker R, Litman F, Kaempf J, Burch K, Schwartz E, Sun S, Payne N. Evaluation and development of potentially better practices to prevent, chronic lung disease and reduce lung injury in neonates. Pediatrics. 2003; 111: e426-31.

20. Brasil. Portaria SAS/MS 31, de 15 de Fevereiro de 1993, trata da presença de neonatologista ou pediatra na sala de parto para assistir o recém-nascido, Diário Oficial da União de 17 de fevereiro de 1993.

21. Sociedade Brasileira de Pediatria. Disponível em: http://www.sbp.com.br. [acesso em 2 nov 2008].

22. Medeiros MLB. Conhecimentos e práticas da displasia broncopulmonar em unidade neonatal pública do município do RJ. [dissertação] Rio de Janeiro: Pós-Graduação em Saúde da Criança e da Mulher do Instituto Fernandes Figueira da Fundação Oswaldo Cruz; 2006.

Recebido em 25 de junho de 2008

Versão final apresentada em 7 de maio de 2009

Aprovado em 8 de junho de 2009
23. Brasil. Ministério da Saúde. Humaniza SUS: política nacional de humanização: documento base para gestores e trabalhadores do SUS 2004. Disponível em: http://bvsms. saude.gov.br/bvs/humanizacao/nov_pub.html

24. Campos GWS. Um método para análise e co-gestão de coletivos: a constituição do sujeito, a produção de valor de uso e a democracia em instituições: o método da roda. São Paulo: Editora Hucitec; 2000.

25. Horbar JD, Plesk PE, Leahy K. NIC/Q 2000: Establishing habits for improvement in neonatal intensive care. Pediatrics. 2003; 111: e397-410.

26. Organização Pan-americana da Saúde (OPAS). Condições de eficiência dos serviços de atenção materno infantil; 1987.

27. Carvalho M, Gomes MASM. A mortalidade do prematuro extremo em nosso meio: realidade e desafios. J Pediatr. (Rio J.) 2005; 81(Sup1 1): S111-8.

28. Menezes DCS, Leite IC, Schramm JMA, Lea MC. Avaliação da peregrinação anteparto numa amostra de puérperas no município do Rio de Janeiro, Brasil, 1999/2001. Cad Saúde Pública. 2006; 22: 553-9. 